retained; a little food, both solid and fluid, taken by the mouth. Urine very scanty and high-coloured.

19th.-Strength increases; but little change in throat since the 16th. Most of the enemata retained; very little food can be swallowed. Spirits improving; voice still thick and nasal. Throat to be swabbed out twice a day with silver solution, sixty grains to the ounce.

24th.- Marked improvement. He sits up for an hour, and is more active and cheerful. No dysphagia; no regurgitation of food; enemata all retained. Back of pharynx presents only a thin slough; posterior part of soft palate merely reddened. 26th.-Moves about the ward. Throat greatly improved, the red base being seen through the grey surface of the pharynx, and the edge of the soft palate looking healthy. Can take most of his food by the mouth, but has still four nutrient enemata daily. Strength of silver solution reduced. Gargle of chlorate of potash ordered. Urine more abundant. 30th. - The slough has entirely disappeared from the pharynx. Strength increased, but three pounds weight said to have been lost within three days.

Oct. 3rd.- Throat now clear. Further loss of two pounds, but strength increased. Slight salivation. Mercurial reduced to one lialf.

7th.-Weight has increased two pounds. Salivation continued. Mercurial inunction stopped.

10th.-Nutrient enemata stopped. Meat diet by the mouth.

14th.--Steady increase in body weight. Still salivation.

17th.-To take syrup of iodide of iron.

21 st.--Has gained two pounds weight in three days. Great improvement in general appearance. Adhesions forming in pharynx, with small points of ulceration.

Nov. 19th.-Discharged cured.

Clinical remarks by Dr. BRUCE.--This case was of considerable interest from its nature, and from a certain degree of difficulty attending its diagnosis; chiefly, however, in connexion with the details of treatment. Active congenital syphilis at the age of thirteen is not so common but that it deserves record, and its diagnosis as a matter of fact may be very far from easy at first. In the present instance the previous personal history and the family history of infantile snuffes were not obtained for several days after admission, and during this time the true nature of the illness was the subject of some discussion. The question of tubercular ulceration was raised, but the absence of swelling of the neck alone went far to dispose of the suggestion. The best immediate evidence of the correctness of the diagnosis of late congenital syphilis was the result of the severe treatment to which the patient was subjected. He was at once put upon mercury. But here a great difficulty arose; the patient could not swallow. Dr. Bruce urged upon the students the absolute necessity of ensuring an abundant supply of nourishment to the tissues whilst a powerful alterative like mercury is being allowed to act upon them. It was contrary to all sound clinical rules to administer mercury to a starving child, and a patient unable to swallow was virtually starving; but just as certainly the patient would shortly die of syphilis. Feeding per oram was therefore given up definitely, and regular nutrient enemata ordered instead, whilst the patient was permitted to attempt to eat occasionally small portions of nutritious food. The method proved successful. A stronger solution of nitrate of silver might have been used from the first for swabbing out the throat, but the shock attending free cauterisation of the whole pharynx might have been too great in the prostrate condition of the patient. As occasionally happens, the rectum, which was irritable at first, came to retain the nutrient enemata very well. There was free salivation for a time, although the mercury was reduced and soon omitted when the mouth became tender. Another curious fact was that the boy continued to lose weight, whilst his general appearance and strength and the local disease were all steadily improving.

\section{DUNDEE ROYAL INFIRMARY.}

ABDOMINAI SANGUINEOUS CYST; ASPIRATION ; RECOVERY; REMARTS

(Under the care of Dr. SrnclaIR.)

J. G--, aged fifty-one, flaxdresser, was admitted on May 14th, 1883, with the following history. About two months previously he observed a smooth round swelling just below the umbilicus, which rapidly increased in size; it caused no discomfort except that arising from its rapidly increasing bulk.
On admission, a rounded swelling, quite smooth to the touch, could be made out in the umbilical region of the abdomen, but extending slightly into the other regions. It was of firm consistence, and appeared to be adherent to the anterior wall of the abdomen (the umbilicus not being movable over it). No fluctuation or impulse could be elicited. There was no bruit on auscultation. The tumour occupied the umbilical part of the epigastric, hypochondriac, and right lumbar regions, and a very small portion of the hypogastric region. It was clearly separable from the hepatic dulness by a narrow resonant line, and it did not move with respiration. The hepatic and splenic dulness were of normal extent; no abnormal signs were discovered in the lungs; the cardiac dulness was normal, but the sounds were weak; tongue slightly furred but moist; appetite good bowels constipated; temperature $99^{\circ}$; pulse 70 , weak; respiration 28; urine pale, slightly acid, sp. gr. 1018, no albumen.

17th.--Hypodermic needle was inserted into tumour, and a syringeful of dark sherry-coloured fluid was readily withdrawn. The fluid contained nearly 50 per cent. of albumen, abundant red blood-corpuscles, and some amorphous masses. No echinococci or hooklets. Sp. gr. 1020.

21 st.-The tumour was aspirated in its centre, and sixty ounces of fluid were withdrawn, of the same character as that removed by the hypodermic syringe.

22nd. - The patient complained of sickness yesterday after the operation. No trace of tumour.

June 2nd.-Patient got up.

6th.--He was dismissed in good general health, the tumour having entirely disappeared.

Remarks by Dr. SINCLAIR.- 1 can find no description of a similar cyst in Bright's classical memoirs. One, in some respects similar, is described by Bauer in Ziemssen's Cyclopædia. ${ }^{1}$ It terminated fatally after two tappings. In THE LaANCET of May 5th, 1883, Dr. Bristowe described two cases of abdominal cysts which resembled the one just described in the rapidity of growth and sanguineous contents. The first he concluded to be probably either a simple cyst of the spleen or a distended kidney, more probably the latter. The second had a much larger history, and was tound on postmortem examination to have been a sanguineous cyst resulting from a round-celled sarcoma. Dr. Clifford Allbutt has described a case ${ }^{2}$ presenting some points of resemblance to mine as regards its contents; but it had a rather higher situation, required three aspirations, re-formed subsequently and then disappeared after an attack of diarrhoea and colic. Dr. Oliver's case, ${ }^{3}$ which occurred in a female, yielded 148 ounces of blood-red fluid on the first, and 84 ounces of similar fluid on the second, tapping, made a much slower recovery than mine, and was considered by him to be probably a hæmatocele of ovarian origin. The case described by my friend, Dr. Reid, and which was watched with great interest by us all, was clearly, 1 think, unconnected with any of the solid viscera of the abdomen. That it was not hydatid was evidenced by the absence of hooklets on careful microscopical examination. I can see no ground for considering its origin malignant. The impression is that it was a cyst of peritoneal origin, possibly owing to a traumatic cause, as the patient is a man of intemperate habits. I saw the man some time ago, a year after dismissal, and found him in good health, and quite capable of performing his usual work; but I thought I discovered some traces of reaccumulation. As cases of this kind are of great interest and rarity, I shall keep a watch on the patient, and report his further history should the disease return.

DOUBLE THORACIC ANEURYSM ; DEATH ; AUTOPSY.

(Under the care of Dr. SinclaIr.)

For the following notes we are indebted to Mr. J. Mankie Whyte, M.A., M.B., house-surgeon.

J.W-, aged forty-nine, a tall well-built man, employed as a labourer in a jute works, was admitted on Feb. 1tth 1884, as an urgent case. IIis history was that he had been subject to pain in the chest since the middle of December 1883, when he fell downstairs while drunk, and received a scalp wound, but no other external injury. The wound healed, but the pain in the chest became worse, catching his breath. For the past fortnight his voice had been hoarse, and he had had a troublesome cough, with inability to expectorate the mucus, which caused a disagreeable rattling noise. Had been in the army; while there he had acquired syphilis; otherwise previous health had been good.

2 The LaxCet, July 7th, 1883. 
On admission, he was in a state of great dyspnoa ; he spoke in a hoarse whisper; cough of a ringing character; throat dry; no difficulty in swallowing; pupils equal; radial pulses equal, 54 per minute, regular; pain in region of heart apex on any exertion; a "gripping" pain, constant, in interscapular region; general præcordial heaving, and epigastric pulsation; apex-beat in fifth space, nipple line; systolic murmur in mitral and aortic areas, loudest in mitral ; second sound accentuated, with a distinct pump-valve character, best marked in aortic area; dulness in upper part of thorax extending from one inch to right of manubrium across sternum for five inches. This dulness was continuous with the cardiac dulness. There was also dulness in the left interscapular region, opposite the spine of the scapula and a little below. The second sound was distinctly heard accentuated over this area. Physical signs of lungs normal, except for occasional bronchitic wheeze. Examination of larynx showed paralysis of left vocal cord. Urine normal. Great improvement followed the treatment, which consisted of rest in bed and administration of bromide and iodide of potassium internally. On May 19th the patient was sent to the Dundee Convalescent Home, but was refused admittance, as he had got drunk on the way.

He presented himself again at the infirmary on Oct. 29th, as bad as ever. Had been employed chiefly in carrying bales of jute; always felt a "gripping" pain in the chest when lifting. Physical signs much the same as when in the infirmary before. Left radial pulse smaller than right, 66 per minute. Improvement was slow, and relapses of dyspnoea occurred. Towards the end of November breathing was very noisy.

On Dec. 10th, about 10.15 A.s., the patient, who had been sleeping quietly, was seized with a paroxysm of dyspnœa. There was laryngeal stridor, like cock-crowing; the face became blue, and he sprang out of bed, saying he was dying. In about five minutes he expired. His face became pale after death.

Autopsy.--Right pleura adherent in whole extent; some tough adhesions also in left, especially near the upper part. Thoracic contents removed in mass with $\operatorname{som} \theta$ difficulty, on account of close adhesions to the vertebræ. There had been no escape of blood from the vessels. Heart empty of blood; both auricles dilated, walls very thin; left ventricle considerably hypertrophied. Aortic valve incompetent; no vegetations but dilatation, circumference being three inches as measured when laid open. Mitral valve somewhat dilated. Ascending part of aorta showed fusiform dilatation, circumference four inches. Wall athoromatous, with ealcareous deposits. The dilatation extended as far as the origin of the left common carotid; the vessels arising from the arch were not themselves involved. Thus the transverse portion towards its termination was of normal calibre, but at the bend it suddenly dilated into an aneurysm larger than a cricket-ball. This consisted of two parts, separated by a constriction; the anterior had the same lining as the aorta, with which it was obviously continuous, the posterior had a wall which was dark in colour and rough, and was largely filled by a laminated organised clot. The pressure of this part had caused deep erosion of the bodies of the third, fourth, and fifth dorsal vertebræ, in front and on the left. The left vagus was traced over the transverse part of the arch and its branch, the left recurrent laryngeal was followed round part of the aneurysm, where it was thickened and irregular, and up alongside the trachea. Neither trachea nor cesophagus showed any effects of pressure; the latter was close to the aneurysm. Lungs much congested; also the abdominal viscera. Fibrous adhesions from old peri-hepatitis all over the liver.

Remarks by Dr. SINCLAIR. - The history of this case clearly suggested aortic aneurysm; the symptoms on admission pointed most distinctly in the same direction; and the physical signs left no doubt that we had before us a case of aneurysm of the arch pressing on the left recurrent laryngeal nerve. In every respect, then, the record was singularly complete and satisfactory up till the moment of the patient's death, which occurred quite suddenly. The autopsy showed that it was not due to rupture of the sac, as might have been anticipated. In the absence of any other quite satisfactory explanation, I suppose paralysis of the heart may be suggested as the most likely cause. The case is interesting also from the fact that there were two distinct aneurysms, separated by a short interval of aorta of normal calibre. This circumstance alone seems to me to be of sufficient interest to justify its being placed on record.

\section{留ledical Sorieties.}

\section{ROYAL MEDICAL AND CHIRURGICAL SOCIETY.}

\section{Discussion on Cholera}

THE adjourned discussion on cholera was held, and the debate concluded, on Tuesday last, Dr. George Johnson, F.R.S., President, in the chair.

Dr. Heneage GrbBes said that he fully agreed with everything that Dr. Klein had said. In acute cases of cholera, instead of a pure cultivation of comma bacilli in the lower part of the ileum, comma bacilli were to be found few and far between. Cultivations of the small straight bacilli in a large number of test tubes proved that these bacilli were not young comma bacilli, but belonged to a distinct species, for they grew in a totally different manner, did not liquefy the gelatine, grew on the surface chiefly, and were not motile. That the comma bacilli were not discovered in the tissues was certainly not due to defective staining. He had used every possible staining fluid and combination of fluids, but had found no comma bacilli, in the intestinal walls. A case of infantile diarrhoea showed a more pronounced morbid change in the intestines than exists in cholera, and so the intestinal lesion could not account for the collapse of cholera. In one case in Lieberkihn's follicles many putrefactive bacilli were present, and this specimen was shown to the Society.

Sir GUYer HuNTER said that, as he understood, Koch had shown that the comma bacillus stood in the relation of cause and effect to cholera. He did not believe that cholera was dependent on the action of a germ. He referred to the characters of the last outbreak of cholera in Egypt and in France as proving his contention. The report on Egyptian cholera presented to Her Majesty's Government also maintained the same views. He considered that cholera had been proved to be non-contagious. A surgeon, writing in the Practitioner of July, 1875, advised the injection of chloral hydrate in doses of 10 grains in 100 minims; this treatment in his hands had been so disastrous that he was compelled to stop it. He did not agree that opiates increased the danger from collapse; any premonitory diarrhoea should be treated by opium and stimulants. He believed cholera to be due to a series or combination of conditions. If the germ is killed by decomposition, then cholera ought not to exist in localities where filth and garbage abound. Strict hygiene was the only way to prevent the occurrence of cholera. Any form of ill-health predisposed to cholera.

Dr. Pye Surrth spoke of the choleraic discharge, and agreed with Cohnheim that this was not of inflammatory origin. In cases of cholera sicca large quantities of detached epithelium were present in the intestines, but this was probably the result of post-mortem change. Moreau obtained a "paralytic secretion" by section of the mesenteric nerves, and the large quantity of effusion thus obtained coincided in many respects with the choleraic discharge. The nervous influence was conveyed, not through the splanchnics or vagus, but by way of the smaller mesenteric ganglia derived from the solar plexus; and excision of these ganglia led to the production of a large intestinal effusion. He was in accord with the generalisation made by Gaskell, to the effect that the exciting influences were conducted by means of grey nerve fibres, whilst inhibitory influences passed along the medullated nerve fibres.

Mr. MaCnamara said that no epidemic was known to have arisen outside the confines of British India. The statements made by Klein with regard to infection were in absolute variance with the conclusions of all previous workers. It was remarkable that the comma bacillus should correspond to the characters of the contagium as described by Parkes-viz., that it was destroyed by acids, that its life in water was very brief, and that the conditions under which the water was placed must be of a certain kind. He referred to the case in which choleraic discharge polluted some water of which nineteen individuals drank, and within thirty-six hours five of these persons were dead of Asiatic cholera. He quoted many circumstances and incidents tending to prove that cholera was a contagious disease. From his observations made on individuals dead of cholera, he could not agree with the views expressed by the President on the existence of spasm of the pulmonary 